\title{
Wissenstransfer ist in der Medizin ein zentraler Aspekt
}

\section{Erwiderung}

Zum Leserbrief von Diehl RR et al. (2020) Unabhängiges Informationsportal? DGNeurologie. https://doi.org/10.1007/s42451-020-00179-5

\section{Originalbeitrag}

Biogen-Anzeige (2019) Unabhängiges Informationsportal - Mit Neurodiem auf dem neuesten Stand. DGNeurologie Ausgabe 5/2019

Gerne möchte ich mich auf den Leserbrief zur Biogen-Anzeige (2019) „Unabhängiges Informationsportal - Mit Neurodiem auf dem neuesten Stand“ aus DGNeurologie Ausgabe 5/2019 beziehen und mich zunächst bei Prof. Dr. Rolf R. Diehl und Kollegen für ihre Ausführungen bedanken. Lassen Sie mich kurz aus meiner Sicht etwas dazu sagen.

Wissenstransfer ist in der Medizin seit jeher ein ganz zentraler Aspekt. Jedoch wird der Informationsfluss schneller, und die Komplexität der Themen steigt. Sich als Arzt auf dem Laufenden $\mathrm{zu}$ halten, wird daher nicht einfacher. Onlineangebote werden für viele Ärzte immer attraktiver, die Reise- und Zeitaufwand einsparen möchten, ohne auf aktuelle Neuigkeiten $\mathrm{zu}$ verzichten. Im Bereich der Neurologie bietet ein Portal wie Neurodiem in Partnerschaft mit führenden Fachjournals wie Nature, JAMA oder The Lancet Wissenstransfer auf qualitativ hohem Niveau. Die inhaltliche Unabhängigkeit ist für uns dabei elementares Kernprinzip. Alle Inhalte werden von externen Dienstleistern und unabhängigen wissenschaftlichen Gremien ausgewählt. Biogen nimmt in keiner Art und Weise Einfluss auf die ausgewählten Inhalte, das ist in unseren Unternehmensrichtlinien auch so festgelegt. Wie in der Forschung wollen wir auch in der digitalen Transformation des Gesundheitswesens neue Wege gehen und aktiv die Zukunft mitgestalten. Objektive, relevante Informationen auf einem modernen Portal wie Neurodiem sollen Neurologen dabei unterstützen, ihre medizinischen und wissenschaftlichen Kenntnisse leichter auf dem aktuellen Stand zu halten, damit sie den Patienten die bestmögliche Versorgung bieten können. Entscheidend ist m. E. letztlich, wie die Ärzte solche digitalen Angebote für sich nutzen möchten und welche Erfahrungen sie dabei machen. Wer ein einfach nutzbares, modernes, werbe- und kostenfreies Informationsportal für neurowissenschaftliche Inhalte schätzt, das auch auf Smartphone und Tablet gut funktioniert, für den könnte sich ein Besuch von www.neurodiem.de lohnen.

\section{Korrespondenzadresse}

\section{Prof. Dr. Andreas Schmitt}

Medical Director Biogen Deutschland

Biogen $\mathrm{GmbH}$

Carl-Zeiss-Ring 6, 85737 Ismaning, Deutschland

andreas.schmitt@biogen.com

Interessenkonflikt. A.Schmitt ist Medical Director der Firma Biogen GmbH. 